\title{
May need more comprehensive approach to residual risks in well controlled hypertensive patients
}

\author{
Toru Suzuki $^{1} \cdot$ Daisuke Kamimura $^{1} \cdot$ Hiromichi Wakui $^{1} \cdot$ Kouichi Tamura $^{1}$
}

Received: 18 September 2020 / Revised: 25 September 2020 / Accepted: 26 September 2020 / Published online: 27 October 2020

(C) The Japanese Society of Hypertension 2020

Several large-scale epidemiological studies, including the Monitoring of Trends and Determinants in Cardiovascular Disease (MONICA) study, showed that during the past few decades, both the incidence of and mortality due to coronary artery disease (CAD) in industrialized countries have decreased [1]. However, CAD is still a leading cause of death in several Western countries, and many patients suffer from this disease worldwide. Although the mortality rate of CAD has been reported to be low in Japan, it is reported that $30-40 \%$ of sudden deaths and $30-50 \%$ of heart failure, which is currently rapidly increasing, are caused by CAD. Furthermore, in recent years, it has been reported that the incidence of CAD in men in urban areas in Japan has been increasing due to impaired glucose tolerance and dyslipidemia caused by obesity and lifestyle changes, including physical inactivity. Therefore, the prevention of CAD is still considered to be an important issue in terms of preventing sudden death, managing quality of life, and contributing to the medical economy.

Hypertension is one of the most critical risk factors for CAD. Globally, in 2015, 1.13 billion adults had hypertension. In Japan, a 2010 survey estimated that there were 43 million hypertensive patients. The Japanese Society of Hypertension (JSH) published the Japanese Society of Hypertension Guidelines for the Management of Hypertension 2019 (JSH 2019) [2]. These guidelines report that only $50 \%$ of hypertensive patients receive treatment, and only $25 \%$ achieve a target blood pressure (BP) level below 140/90 mmHg. A previously published large-scale epidemiological study performed in Japan showed that the incidence rate of CAD was lowest in those with optimal blood

Kouichi Tamura

tamukou@med.yokohama-cu.ac.jp

1 Department of Medical Science and Cardiorenal Medicine, Yokohama City University Graduate School of Medicine, Yokohama, Japan pressure (systolic blood pressure $<120 \mathrm{mmHg}$ and diastolic blood pressure $<80 \mathrm{mmHg}$ ), and the risk increased with increasing blood pressure (Fig. 1) [3]. Additionally, in the Systolic Blood Pressure Intervention Trial (SPRINT), which was performed worldwide, the incidence of cardiovascular disease was decreased in the intensive antihypertensive treatment group (systolic blood pressure $<120 \mathrm{mmHg}$ ) compared with the standard hypertensive treatment group (systolic blood pressure $<140 \mathrm{mmHg}$ ) [4]. Therefore, the JSH 2019 guidelines as well as the most recent American College of Cardiology/American Heart Association guidelines recommend strict blood pressure management [5].

In this issue of Hypertension Research, Tsukihashi et al. analyzed a large number of coronary computed tomography angiography (CCTA) data to investigate the presence and severity of CAD in the intensive blood pressure-lowering therapy group compared to those in the nonhypertensive group [6]. The authors showed that the intensive blood pressure-lowering group had a higher prevalence and more severe CAD than the nonhypertensive group. In the logistic

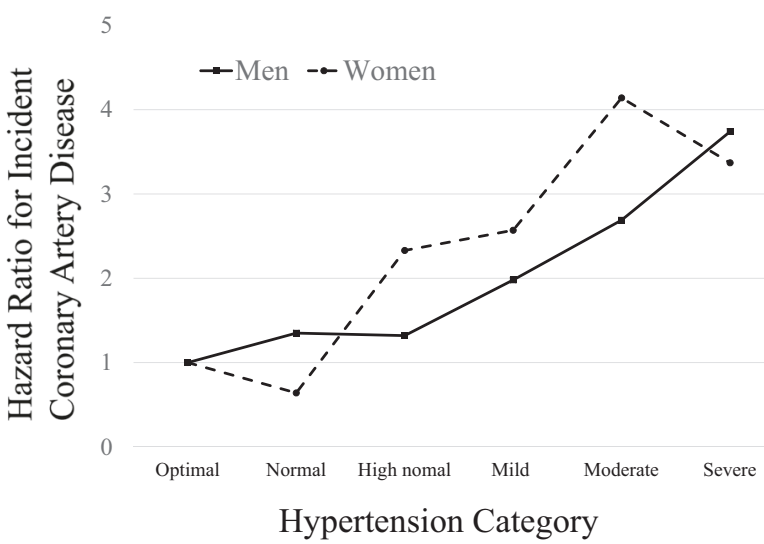

Fig. 1 Hazard ratios for incident coronary artery disease according to the hypertension categories based on data from the JPHC Study [3]. Men: adjusted for age, region, body mass index, smoking, alcohol consumption, anti-hypertensive medication, diabetes, and total cholesterol. Women: adjusted for age 
regression analysis, after adjustment for possible confounding factors, the intensive blood pressure-lowering group still had a higher prevalence of CAD. There are some limitations of this study, as the duration of antihypertensive treatment in the intensive blood pressure-lowering group received was not reported, and the frequency of symptomatic patients in each group was not clarified. Therefore, possible or unclarified confounding factors might not have been fully adjusted for. The important insight from this study is that many patients with well-controlled hypertension do not exhibit clinical CAD symptoms; however, these patients may need more intensive care for residual coronary risks.

There are several well-documented coronary risk factors, including dyslipidemia, diabetes mellitus, smoking, obesity and physical inactivity. It is well established that plasma low-density lipoprotein (LDL) cholesterol levels predict cardiovascular event rates in patients with documented CAD and that LDL lowering, whether by statins or by ezetimibe, reduces this risk [7]. Newly developed PCSK9 inhibitors, including alirocumab, evolocumab and bococizumab, could provide additional risk reduction for future coronary events [8]. Diabetes is also a major risk factor for CAD [9]. However, in the Action to Control Cardiovascular Risk in Diabetes (ACCORD) trial, compared with standard therapy, the use of intensive therapy to target normal glycated hemoglobin levels for 3.5 years increased mortality and did not significantly reduce the occurrence of major cardiovascular events [10]. Thus, reducing blood glucose levels may not lead to a reduction in future CAD events. Sodium glucose cotransporter (SGLT) 2 inhibitors are a new class of blood glucose-lowering agents, and several clinical trials have shown their potential to reduce CAD events and heart failure; however, the mechanisms of these associations have not yet been well clarified [11]. Cigarette smoking is also an independent risk factor for the development of CAD. Smoking causes inflammation, endothelial dysfunction, platelet dysfunction, and increased oxidative stress, all of which are associated with the development of CAD. The proportions of current smokers in developed countries is currently 10 to $30 \%$, and the number of smokers has been increasing in developing countries. Thus, quitting smoking is important to prevent the development of CAD. Physical inactivity is another risk factor for CAD. Many previous reports have suggested that habitual physical activity is associated with a decreased prevalence of coronary artery calcification and a reduced risk of CAD. Physical activity reduces systemic inflammation, improves endothelial function, and decreases oxidative stress, and these effects suppress the development of CAD [12]. New approaches for preventing CAD, such as anti-inflammatory therapies (colchicine) or genome-guided approaches that are able to provide more personalized prevention strategies, have been attempted, and some have been reported to be effective [13, 14].

As noted above, the morbidity of and mortality due to CAD have been decreasing in industrialized countries. The factors contributing to decreased mortality are mainly related to prevention strategies, namely, optimal medical therapy and improving modifiable lifestyle habits. Thus, from the results of the study by Tsukihashi et al., even if blood pressure is well controlled, it is still necessary to rigorously enforce relevant medical therapy, encourage patients to improve modifiable lifestyle habits and incorporate new preventive strategies into daily clinical practice [15].

\section{Compliance with ethical standards}

Conflict of interest The authors declare that they have no conflict of interest.

Publisher's note Springer Nature remains neutral with regard to jurisdictional claims in published maps and institutional affiliations.

\section{References}

1. Tunstall-Pedoe H, Kuulasmaa K, Mahonen M, Tolonen H, Ruokokoski E, Amouyel P. Contribution of trends in survival and coronary-event rates to changes in coronary heart disease mortality: 10-year results from 37 WHO MONICA project populations. Monitoring trends and determinants in cardiovascular disease. Lancet 1999;353:1547-57.

2. Umemura S, Arima H, Arima S, Asayama K, Dohi Y, Hirooka Y, et al. The Japanese Society of Hypertension Guidelines for the Management of Hypertension (JSH 2019). Hypertens Res. 2019;42:1235-481.

3. Ikeda A, Iso H, Yamagishi K, Inoue M, Tsugane S. Blood pressure and the risk of stroke, cardiovascular disease, and all-cause mortality among Japanese: the JPHC Study. Am J Hypertens. 2009;22:273-80.

4. Wright JT Jr, Williamson JD, Whelton PK, Snyder JK, Sink KM, Rocco MV, et al. A randomized trial of intensive versus standard blood-pressure control. N. Engl J Med. 2015;373:2103-16.

5. Whelton PK, Carey RM, Aronow WS, Casey DE Jr, Collins KJ, Himmelfarb CD, et al. 2017 ACC/AHA/AAPA/ABC/ACPM/ AGS/APhA/ASH/ASPC/NMA/PCNA Guideline for the Prevention, Detection, Evaluation, and Management of High Blood Pressure in Adults: A Report of the American College of Cardiology/American Heart Association Task Force on Clinical Practice Guidelines. Hypertension 2018;71:e13-115.

6. Tsukihashi Y, Shiga Y, Suematsu Y, Idemoto Y, Tashiro K, Yano $\mathrm{Y}$, et al. Presence and severity of coronary artery disease in patients who achieved intensive blood pressure reduction at the time of coronary computed tomography angiography. Hypertens Res. 2020. https://doi.org/10.1038/s41440-020-00545-6.

7. Cannon CP, Blazing MA, Giugliano RP, Amy MC, White JA, Pierre T, et al. Ezetimibe Added to Statin Therapy after Acute Coronary Syndromes. N. Engl J Med. 2015;372:2387-97.

8. Rosenson RS, Hegele RA, Fazio S, Cannon CP. The Evolving Future of PCSK9 Inhibitors. J Am Coll Cardiol. 2018;72:314-29.

9. Gerstein HC, Miller ME, Byington RP, Goff DC Jr, Bigger JT, Buse JB, et al. Effects of intensive glucose lowering in type 2 diabetes. N. Engl J Med. 2008;358:2545-59. 
10. Zinman B, Wanner C, Lachin JM, Fitchett D, Bluhmki E, Hantel S, et al. Empagliflozin, Cardiovascular Outcomes, and Mortality in Type 2 Diabetes. N. Engl J Med. 2015;373:2117-28.

11. Winzer EB, Woitek F, Linke A Physical Activity in the Prevention and Treatment of Coronary Artery Disease. J Am Heart Assoc. 2018;7.

12. Kai H, Katoh A, Harada H, Niiyama H, Furukawa Y, Kimura T. Low blood pressure and cardiovascular events in diabetic patients with coronary artery disease after revascularization: the CREDO-Kyoto registry cohort-1. Hypertens Res. 2020; 43:715-23.
13. Nidorf SM, Fiolet ATL, Eikelboom JW, Astrid S, Opstal TSJ, Bax WA, et al. The effect of low-dose colchicine in patients with stable coronary artery disease: The LoDoCo2 trial rationale, design, and baseline characteristics. Am Heart J. 2019;218:46-56.

14. Steg PG, Ducrocq G. Future of the Prevention and Treatment of Coronary Artery Disease. Circ J. 2016;80:1067-72.

15. Picard F, Panagiotidou P, Wolf-Pütz A, Buschmann I, Buschmann $\mathrm{E}$, Steffen M, et al. Individual shear rate therapy (ISRT)-further development of external counterpulsation for decreasing blood pressure in patients with symptomatic coronary artery disease (CAD). Hypertens Res. 2020;43:186-96. 